\title{
KIC 1718290: A Helium-Rich V1093-Her-like Pulsator on the Blue Horizontal Branch
}

\author{
R. H. Østensen \\ P. Degroote \\ J. H. Telting \\ J. Vos \\ C. Aerts
}

See next page for additional authors

Follow this and additional works at: https://bearworks.missouristate.edu/articles-cnas

\section{Recommended Citation}

Østensen, Roy H., Pieter Degroote, John H. Telting, Joris Vos, Conny Aerts, C. Simon Jeffery, Elizabeth M. Green, Mike D. Reed, and Ulirich Heber. "KIC 1718290: A Helium-Rich V1093-Her-like Pulsator on the Blue Horizontal Branch." The Astrophysical Journal Letters 753, no. 1 (2012): L17.

This article or document was made available through BearWorks, the institutional repository of Missouri State University. The work contained in it may be protected by copyright and require permission of the copyright holder for reuse or redistribution.

For more information, please contact BearWorks@library.missouristate.edu. 


\section{Authors}

R. H. Østensen, P. Degroote, J. H. Telting, J. Vos, C. Aerts, C. S. Jerrery, E. M. Green, Michael D. Reed, and U. Heber 


\title{
KIC 1718290: A HELIUM-RICH V1093-Her-LIKE PULSATOR ON THE BLUE HORIZONTAL BRANCH
}

\author{
R. H. Østensen ${ }^{1}$, P. Degroote ${ }^{1}$, J. H. Telting ${ }^{2}$, J. $\operatorname{Vos}^{1}$, C. Aerts ${ }^{1,3}$, C. S. Jefferr ${ }^{4}$, E. M. Green ${ }^{5}$, \\ M. D. $\operatorname{ReED}^{6}$, AND U. HeBER ${ }^{7}$ \\ ${ }^{1}$ Instituut voor Sterrenkunde, K.U. Leuven, Celestijnenlaan 200D, B-3001 Leuven, Belgium; roy@ ster.kuleuven.be \\ 2 Nordic Optical Telescope, Apartado 474, 38700 Santa Cruz de La Palma, Spain \\ ${ }^{3}$ Department of Astrophysics, IMAPP, Radboud University Nijmegen, 6500 GL Nijmegen, The Netherlands \\ ${ }^{4}$ Armagh Observatory, College Hill, Armagh BT61 9DG, UK \\ 5 Steward Observatory, University of Arizona, 933 N. Cherry Avenue, Tucson, AZ 85721, USA \\ ${ }^{6}$ Department of Physics, Astronomy, and Materials Science, Missouri State University, Springfield, MO 65897, USA \\ ${ }^{7}$ Dr. Karl Remeis-Observatory \& ECAP, Astronomisches Inst., FAU Erlangen-Nuremberg, Sternwartstr. 7, D-96049 Bamberg, Germany \\ Received 2012 April 25; accepted 2012 May 30; published 2012 June 15
}

\begin{abstract}
We introduce the first $g$-mode pulsator found to reside on the classical blue horizontal branch. One year of Kepler observations of KIC 1718290 reveals a rich spectrum of low-amplitude modes with periods between 1 and $12 \mathrm{hr}$, most of which follow a regular spacing of $276.3 \mathrm{~s}$. This mode structure strongly resembles that of the V1093 Her pulsators, with only a slight shift toward longer periods. Our spectroscopy, however, reveals KIC 1718290 to be quite distinct from the sdB stars that show V1093 Her pulsations, which all have surface gravities higher than $\log g=5.1$ and helium abundances depleted by at least an order of magnitude relative to the solar composition. We find that KIC 1718290 has $T_{\text {eff }}=22,100 \mathrm{~K}, \log g=4.72$, and a super-solar helium abundance ( $\log N_{\mathrm{He}} / N_{\mathrm{H}}=-0.45$ ). This places it well above the extreme horizontal branch, rather on the very blue end of the classical horizontal branch, where shell hydrogen burning is present. We conclude that KIC 1718290 must have suffered extreme mass loss during its first giant stage, but not sufficient to reach the extreme horizontal branch.
\end{abstract}

Key words: stars: horizontal-branch - stars: individual (KIC 1718290) - stars: oscillations - stars: variables: general - subdwarfs

Online-only material: color figures

\section{INTRODUCTION}

The Kepler spacecraft is monitoring a $105 \mathrm{deg}^{2}$ field in the Cygnus-Lyrae region, primarily to detect transiting planets (Borucki et al. 2011). As a bycatch, pulsating stars are observed, and these high-quality data sets are a treasure trove for asteroseismology studies (Gilliland et al. 2010). In the first four quarters of the Kepler Mission, a survey for pulsating stars was made, and a total of 113 compact-pulsator candidates were checked for variability (Østensen et al. 2010c, 2011b). The survey was extremely successful with respect to subdwarf-B (sdB) pulsators, with discoveries including one clear V361 Hya pulsator (Kawaler et al. 2010b), a total of thirteen V1093 Her stars (Reed et al. 2010; Kawaler et al. 2010a; Baran et al. 2011; Østensen et al. 2010a).

Preselection of Kepler targets based on their photometric and spectroscopic properties has provided us with the most outstanding photometric light curves in the history of astronomy for a host of known pulsator types. In addition, the large archive of Kepler data, ${ }^{8}$ mostly obtained to detect exoplanets, provides an inexhaustible source of spectacular light curves of all kinds of variable stars, and unlimited opportunities for exciting new discoveries. Here we present one such. It was included in the color-selected sample of white-dwarf-pulsator candidates that provided the only V777 Her pulsator in the Kepler field (KIC 8626021; Østensen et al. 2011a). However, the spectrum obtained in that survey placed it well outside of the known pulsator ranges, and it was not considered further. By chance, it was observed by Kepler as an exoplanet target, permitting

\footnotetext{
8 http://archive.stsci.edu/kepler/
}

serendipity to trump our otherwise pinpoint-precision survey strategy.

Our spectrum shows that KIC 1718290 is technically a hot subdwarf of the sdB type, as it appears as a B-type star with a rather high surface gravity, placing it below the main sequence in the Hertzsprung-Russell diagram. However, its surface gravity is lower and its helium abundance is much higher than the majority of the sdBs, making it quite unusual.

After ascending the red giant branch (RGB), most stars with main-sequence masses less than $\sim 2 M_{\odot}$ will undergo a corehelium flash and end up by the red clump, where they spend $\sim 100 \mathrm{Myr}$ burning helium in the core, before ascending the asymptotic giant branch (AGB). If such stars suffer significant mass loss, then they will appear bluer after helium ignition while still having roughly the same luminosity. This produces the distinctive feature seen in color-magnitude diagrams of globular clusters, known as the horizontal branch (HB). HB stars possess a hydrogen-burning shell, whenever the hydrogen envelope contains $>0.02 M_{\odot}$. Stars with thinner envelopes will reside on the extreme horizontal branch (EHB; Heber 1986). Other HB stars are often designated as either red or blue (RHB or BHB), with RHB stars encompassing the red clump and the notable class of RR Lyrae pulsators. These are classical radial pulsators excited by the same heat mechanism acting in the partial ionization zone of $\mathrm{He}^{+} / \mathrm{He}^{2+}$ that also drives pulsations in Cepheids and $\delta$ Scuti stars (Christy 1966). The BHB stars include all stars of spectral type A and B up to the start of the EHB at $\log g=5$. The distribution of stars along the HB is a convoluted function of mass loss on the RGB, overall metallicity, and interactions with a close companion.

The process that produces the EHB stars is thought to include a large contribution from binaries that underwent mass transfer 

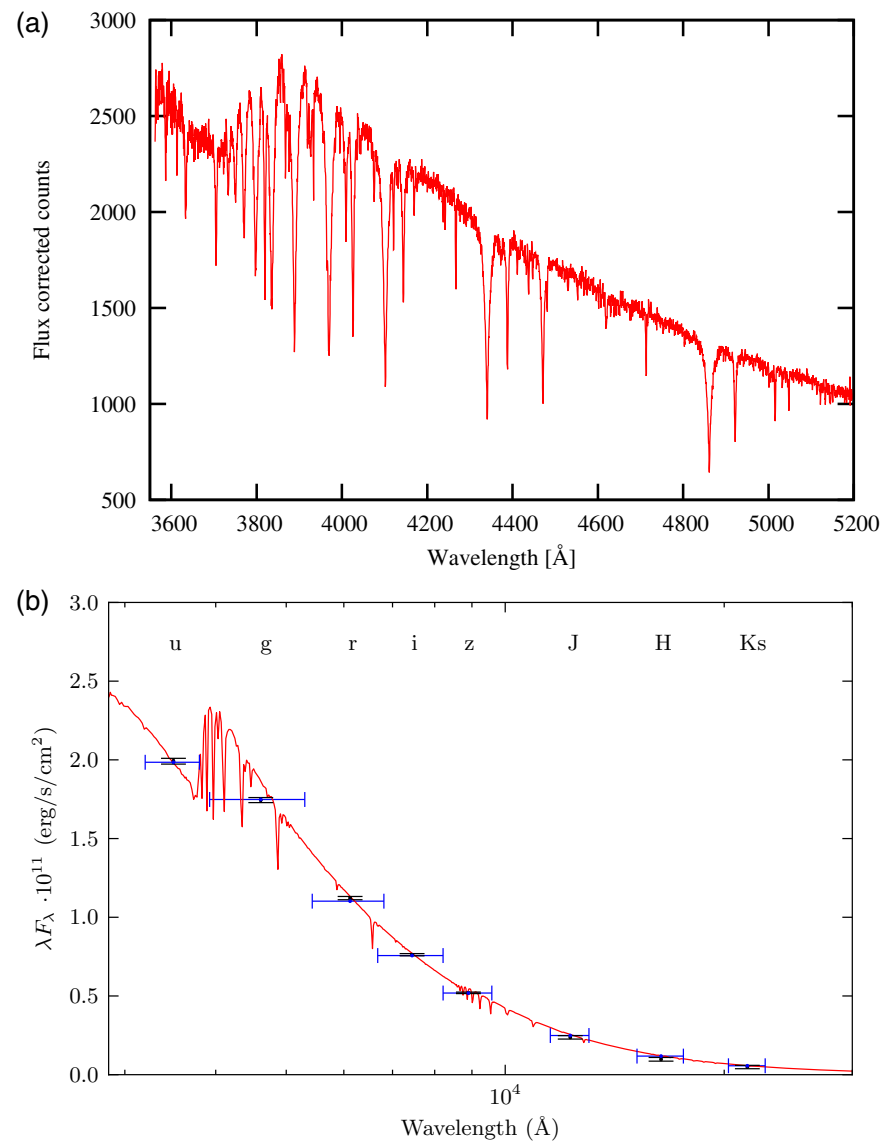

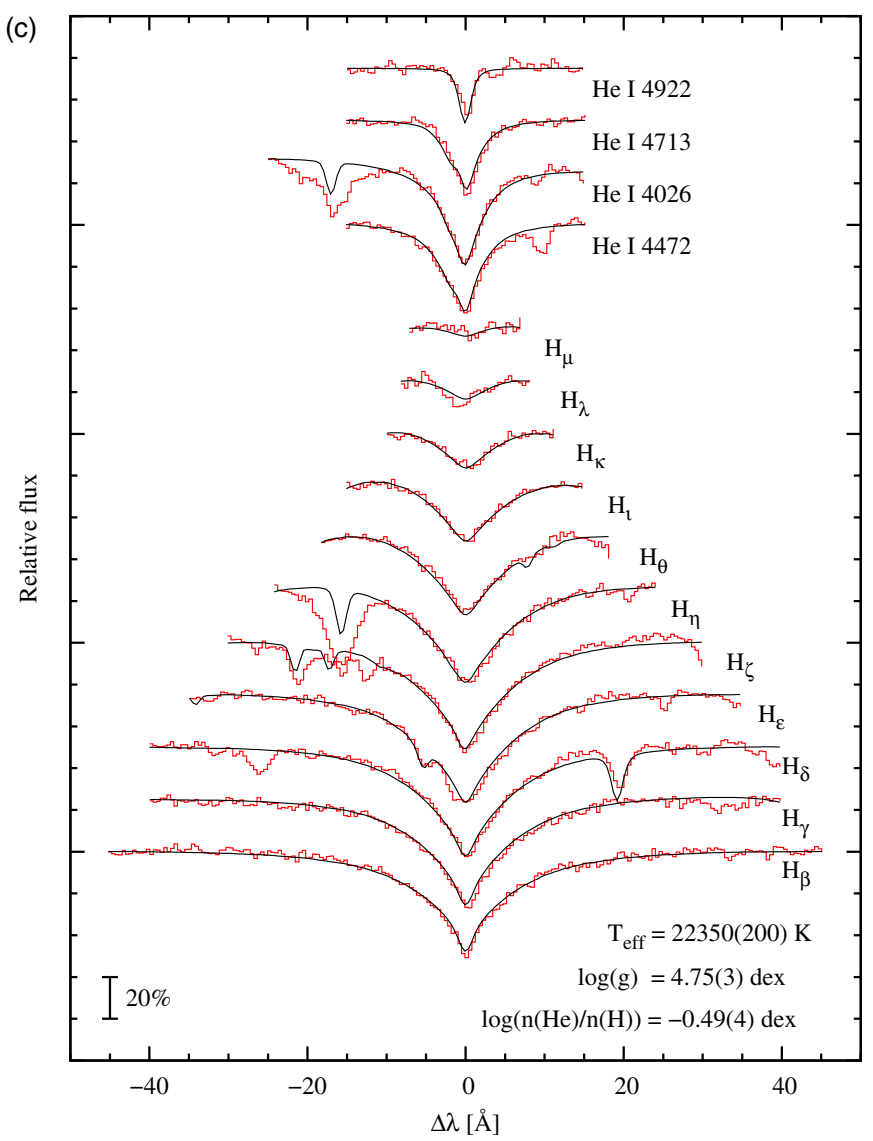

Figure 1. (a) The discovery spectrum of KIC 1718290. (b) SED fit to broadband photometry. (c) Line-profile fit to the WHT spectrum. (A color version of this figure is available in the online journal.)

and drastic mass loss in either common-envelope or Roche-lobeoverfilling configurations on the RGB (Han et al. 2002, 2003). Whereas the majority of the sdB stars on the EHB are members of short-period binaries (Østensen 2009 and references therein), such short-period binaries are hard to find on the classical HB (Prša et al. 2008).

Since the discovery of short-period pulsations by Kilkenny et al. (1997), the sdB stars have been extensively studied. The $p$-mode pulsators are known as V361 Hya stars after the prototype, and constitute $\sim 10 \%$ of sdBs with temperatures between $\sim 28,000 \mathrm{~K}$ and 36,000 K (Østensen et al. 2010b). Green et al. (2003) discovered longer-period pulsations in $75 \%$ of stars between $\sim 22,000 \mathrm{~K}$ and 30,000 K: the V1093 Her stars. Here we present the first discovery of a star that exhibits longperiod pulsations similar to the V1093 Her stars on the EHB, but is located on the BHB instead.

\section{TARGET PROPERTIES}

KIC $1718290(K p=15.486)$ was first noted to be a UVexcess star from Sloan Digital Sky Survey (SDSS) photometry (Stoughton et al. 2002). It appears as an exceedingly blue object $^{9} 45^{\prime \prime}$ to the east of BD $+36^{\circ} 3529$. The SDSS database lists it as SDSS J192300.68+371504.4 with ugriz = 15.505(4), 15.293(3), 15.481(4), 15.687(4), 15.919(7). The $u-g$ color term of +0.21 placed it above the red cutoff of our initial

9 http://cas.sdss.org/dr7/en/tools/chart/navi.asp? $\mathrm{ra}=290.753 \& \mathrm{dec}=37.251$ survey, but in the 2010 search we allowed more generous margins to catch possible ZZ-Ceti pulsators and sdBVs reddened by dust, as is the case with KIC 1718290. The target also appears as 2MASS J19230068+3715044 with $J H K_{s}=$ 15.50(5), 15.71(13), 15.69(24).

We used the atmosphere models of Kurucz (1993) to perform a fit to the broadband photometry following the procedure in Degroote et al. (2011). The fit is consistent with a $T_{\text {eff }}=$ $21,000(1000) \mathrm{K}$, and $E(B-V)=0.192(8)$, but provides no useful constraint on the surface gravity (Figure 1(b)). The optimal reddening agrees with Schlegel et al. (1998), and we conclude that the rather red color is caused by dust and not a composite-spectrum object.

We obtained a classification spectrum of KIC 1718290 with the ISIS spectrograph on the $4.2 \mathrm{~m}$ William Herschel Telescope (WHT) on 2010 July 3, using the R600B grating $(R=1.7 \AA$ AWHM $)$, giving $\mathrm{S} / \mathrm{N} \sim 45 \mathrm{pixel}^{-1}$. Over 28 days in 2012 March/April we obtained a further five $\mathrm{S} / \mathrm{N} \sim 50$ spectra with the Nordic Optical Telescope (NOT) using ALFOSC with grism $16(R=2.2 \AA \mathrm{FWHM})$. The mean radial velocity (RV) is $-29.2 \mathrm{~km} \mathrm{~s}^{-1}$, with a standard deviation of $7.5 \mathrm{~km} \mathrm{~s}^{-1}$, demonstrating that low-amplitude RV variability might be present. The coverage is insufficient to rule out binarity at periods around a month or longer.

Standard spectral extraction and wavelength calibration was done using IRAF. The spectrum shown in Figure 1(a) is the WHT observation after adjusting the slope by an instrument response function derived from an observation of Feige 67 obtained on the same night. 


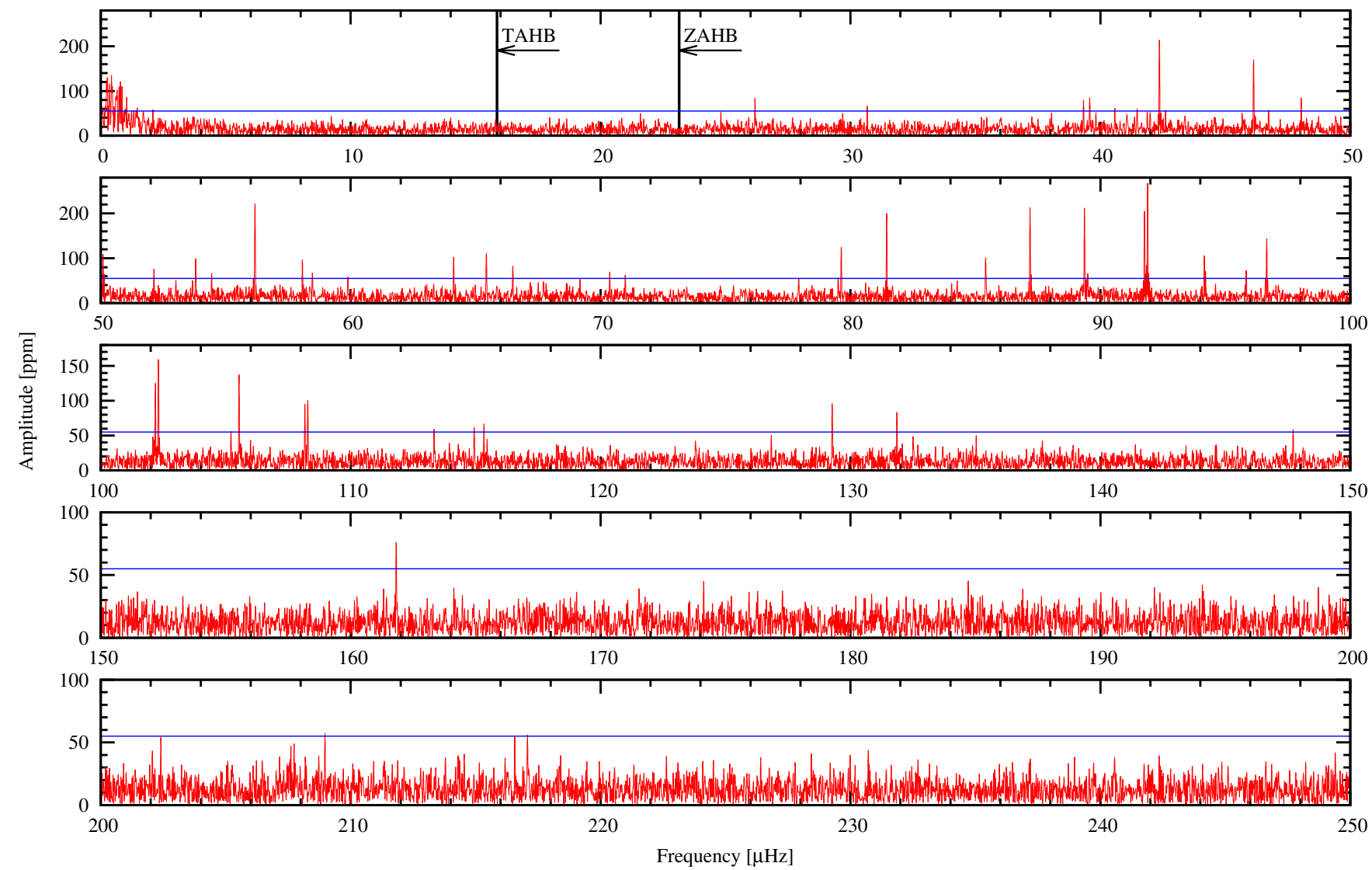

Figure 2. FT of the Kepler light curve of KIC 1718290, including data from Q1 to Q5. The $4 \sigma_{\mathrm{FT}}$ level is indicated with a horizontal line. Vertical bars indicate the range for the theoretical lower limit for $g$-modes on the ZAHB and TAHB, respectively.

(A color version of this figure is available in the online journal.)

A fit to the spectra on an LTE grid suitable for these stars (see Heber et al. 2000; Ramspeck et al. 2001 for details) yielded an effective temperature of $T_{\text {eff }}=22,350(200) \mathrm{K}$, a surface gravity of $\log g=4.75(3)$, and a helium abundance of $\log N_{\mathrm{He}} / N_{\mathrm{H}}=$ -0.49 (4) from the WHT spectrum (Figure 1(c)). The mean NOT spectrum yields $T_{\text {eff }}=21,796(144) \mathrm{K}, \log g=4.67(3)$, and $\log N_{\mathrm{He}} / N_{\mathrm{H}}=-0.40(4)$.

These parameters place KIC 1718290 well above the red, low-gravity edge of the EHB in the $T_{\text {eff }}-\log g$ plane, indicating that it could still have sufficient envelope mass to sustain a weak hydrogen-burning shell.

\section{FREQUENCY ANALYSIS}

We analyzed the Kepler photometry both from pipelineextracted light curves and pixel data. As the star is only $\sim 10$ pixels away from the much brighter star $\mathrm{BD}+36^{\circ} 3529$, some contamination is present. The Kepler target catalog lists it with contamination factors of $8.8 \%, 11.6 \%, 13.6 \%$, and $6.6 \%$, depending on quarter. We explored the background pixels surrounding the target in the pixel apertures to see if any trace of a pulsation signature could originate from $\mathrm{BD}+36^{\circ} 3529$, but found no significant signal in the frequency range where KIC 1718290 displays its features. After extracting the longcadence light curves from quarters 1 through 5 , we subtracted out the slow trends introduced by the varying contamination from the neighboring star with low-order polynomials and rapidly decaying exponentials for the leading slope that often occurs during the first days after the spacecraft has reacquired the field. The light curve commences at BJD 2454964.51194, ends at BJD 2455371.16248, and spans 406.6 days. The monthly gaps are typically about 1 day, with the largest gap being 5 days. The Fourier transform (FT) of this light curve is shown in Figure 2. A rich spectrum of low-amplitude peaks can be easily discerned, starting at $\sim 25 \mu \mathrm{Hz}$ and extending up to $217 \mu \mathrm{Hz}$. The straight lines in Figure 2 indicate the $4 \sigma_{\mathrm{FT}}$ level of $54.8 \mathrm{ppm}$. We consider peaks of less than $4.2 \sigma_{\mathrm{FT}}$ or $58 \mathrm{ppm}$ as tentative indications, as we have seen spurious detections at the $4.1 \sigma_{\mathrm{FT}}$ level in many other Kepler targets (Østensen et al. 2010c).

The longest periods seen in KIC 1718290 are significantly longer than those seen in the stars of Reed et al. (2011). So, we computed the cutoff frequency for $g$-modes according to the prescription of Hansen et al. (1985) and found that the longest period that can be sustained is $\sim 0.5$ days at the zero-age $\mathrm{HB}$ and peaks at $\sim 0.73$ days close to the terminal-age HB when using the $0.476 M_{\odot}$ model described in the next section. In Figure 2 the cutoff frequency range is marked with vertical bars.

The frequencies and periods of the detected peaks are listed in Table 1, together with their amplitudes and phases relative to the first data point. The resolution of the data set is $\sim 0.03 \mu \mathrm{Hz}$, and can be taken as the error on the measured frequencies. We detected 54 frequencies, 8 of which are close pairs. No triplets or higher-order multiplets are seen. All of the frequencies except for nine can be prewhitened out of the light curve, demonstrating that they have stable amplitudes. A few of the peaks may also be combination frequencies.

The most discernible feature of this list is the regular sequence of peaks with an even period spacing of $\Delta \Pi_{1}=276.3 \mathrm{~s}$. This 
Table 1

List of Frequencies

\begin{tabular}{|c|c|c|c|c|c|c|c|}
\hline ID & $\begin{array}{l}\text { Freq. } \\
(\mu \mathrm{Hz})\end{array}$ & $\begin{array}{c}P \\
\text { (days) }\end{array}$ & $\begin{array}{c}A \\
(\mathrm{ppm})\end{array}$ & $\begin{array}{l}\text { Phase } \\
\text { (cyc) }\end{array}$ & $n_{\ell=1}$ & $n_{\ell=2}$ & Note \\
\hline$\overline{f_{1}}$ & 24.81 & 0.4665 & 54 & 0.290 & & & $\mathrm{t}$ \\
\hline$f_{2}$ & 26.18 & 0.4421 & 84 & 0.030 & & & $\mathrm{u}$ \\
\hline$f_{3}$ & 30.67 & 0.3774 & 67 & 0.503 & & & $\mathrm{u}$ \\
\hline$f_{4}$ & 39.32 & 0.2943 & 79 & 0.812 & $91 \dagger$ & & d \\
\hline$f_{5}$ & 39.57 & 0.2925 & 82 & 0.864 & 91 & & d \\
\hline$f_{6}$ & 40.58 & 0.2852 & 58 & 0.588 & $89 \dagger$ & & $\mathrm{t}$ \\
\hline$f_{7}$ & 41.47 & 0.2791 & 56 & 0.758 & $87 \dagger$ & 151 & $\mathrm{t}$ \\
\hline$f_{8}$ & 41.88 & 0.2764 & 57 & 0.290 & 86 & & \\
\hline$f_{9}$ & 42.36 & 0.2732 & 214 & 0.635 & 85 & & \\
\hline$f_{10}$ & 46.13 & 0.2509 & 171 & 0.357 & 78 & & \\
\hline$f_{11}$ & 46.73 & 0.2477 & 57 & 0.768 & 77 & 134 & \\
\hline$f_{12}$ & 48.04 & 0.2409 & 88 & 0.980 & 75 & & \\
\hline$f_{13}$ & 50.09 & 0.2311 & 111 & 0.939 & & 125 & \\
\hline$f_{14}$ & 52.13 & 0.2220 & 74 & 0.381 & 69 & 120 & \\
\hline$f_{15}$ & 53.80 & 0.2151 & 101 & 0.539 & $67 \dagger$ & & \\
\hline$f_{16}$ & 54.44 & 0.2126 & 70 & 0.504 & 66 & 115 & $\mathrm{p}$ \\
\hline$f_{17}$ & 56.17 & 0.2061 & 222 & 0.248 & 64 & & \\
\hline$f_{18}$ & 58.07 & 0.1993 & 96 & 0.321 & $62 \dagger$ & & \\
\hline$f_{19}$ & 58.47 & 0.1980 & 68 & 0.611 & & 107 & \\
\hline$f_{20}$ & 59.89 & 0.1933 & 58 & 0.333 & 60 & & $\mathrm{t}$ \\
\hline$f_{21}$ & 64.12 & 0.1805 & 103 & 0.099 & 56 & & $\mathrm{c}$ \\
\hline$f_{22}$ & 65.43 & 0.1769 & 111 & 0.309 & $55 \dagger$ & & \\
\hline$f_{23}$ & 66.49 & 0.1741 & 80 & 0.102 & 54 & 94 & $\mathrm{p}$ \\
\hline$f_{24}$ & 70.36 & 0.1645 & 70 & 0.888 & 51 & & \\
\hline$f_{25}$ & 70.99 & 0.1630 & 61 & 0.639 & & 88 & \\
\hline$f_{26}$ & 77.93 & 0.1485 & 56 & 0.462 & 46 & & $\mathrm{t}$ \\
\hline$f_{27}$ & 79.51 & 0.1456 & 55 & 0.159 & 45 & & $\mathrm{t}, \mathrm{d}, \mathrm{p}$ \\
\hline$f_{28}$ & 79.63 & 0.1454 & 125 & 0.364 & 45 & & $\mathrm{~d}$ \\
\hline$f_{29}$ & 81.45 & 0.1421 & 201 & 0.026 & 44 & & $\mathrm{c}$ \\
\hline$f_{30}$ & 85.41 & 0.1355 & 100 & 0.097 & 42 & 73 & \\
\hline$f_{31}$ & 87.19 & 0.1328 & 214 & 0.879 & $41 \dagger$ & & \\
\hline$f_{32}$ & 89.36 & 0.1295 & 213 & 0.474 & 40 & & $\mathrm{~d}$ \\
\hline$f_{33}$ & 89.49 & 0.1293 & 73 & 0.639 & 40 & & $\mathrm{c}, \mathrm{d}$ \\
\hline$f_{34}$ & 91.77 & 0.1261 & 205 & 0.994 & 39 & 68 & $\mathrm{~d}$ \\
\hline$f_{35}$ & 91.89 & 0.1260 & 268 & 0.129 & 39 & & $\mathrm{~d}$ \\
\hline$f_{36}$ & 94.16 & 0.1229 & 103 & 0.805 & 38 & & $\mathrm{c}, \mathrm{d}$ \\
\hline$f_{37}$ & 94.21 & 0.1229 & 57 & 0.955 & 38 & & $\mathrm{t}, \mathrm{d}$ \\
\hline$f_{38}$ & 95.84 & 0.1208 & 74 & 0.613 & & 65 & $\mathrm{p}$ \\
\hline$f_{39}$ & 96.66 & 0.1198 & 144 & 0.879 & 37 & & \\
\hline$f_{40}$ & 102.19 & 0.1133 & 125 & 0.083 & 35 & & d \\
\hline$f_{41}$ & 102.31 & 0.1131 & 160 & 0.521 & 35 & & $\mathrm{c}, \mathrm{d}$ \\
\hline$f_{42}$ & 105.53 & 0.1097 & 138 & 0.236 & $34 \dagger$ & 59 & \\
\hline$f_{43}$ & 108.17 & 0.1070 & 96 & 0.181 & 33 & & $\mathrm{c}, \mathrm{d}$ \\
\hline$f_{44}$ & 108.28 & 0.1069 & 101 & 0.294 & 33 & & $\mathrm{c}, \mathrm{d}$ \\
\hline$f_{45}$ & 113.34 & 0.1021 & 58 & 0.183 & & $55 \dagger$ & $\mathrm{c}, \mathrm{t}$ \\
\hline$f_{46}$ & 114.94 & 0.1007 & 61 & 0.919 & 31 & $54 \dagger$ & $\mathrm{d}$ \\
\hline$f_{47}$ & 115.33 & 0.1004 & 68 & 0.855 & 31 & 54 & $\mathrm{~d}$ \\
\hline$f_{48}$ & 129.27 & 0.0895 & 96 & 0.749 & & 48 & \\
\hline$f_{49}$ & 131.86 & 0.0878 & 83 & 0.770 & 27 & & $\mathrm{c}, \mathrm{p}$ \\
\hline$f_{50}$ & 147.71 & 0.0784 & 58 & 0.768 & 24 & 42 & $\mathrm{c}, \mathrm{p}$ \\
\hline$f_{51}$ & 161.82 & 0.0715 & 76 & 0.954 & 22 & & \\
\hline$f_{52}$ & 208.97 & 0.0554 & 57 & 0.323 & $17 \dagger$ & & $\mathrm{t}, \mathrm{p}$ \\
\hline$f_{53}$ & 216.57 & 0.0534 & 54 & 0.084 & & & $\mathrm{c}, \mathrm{t}, \mathrm{p}$ \\
\hline$f_{54}$ & 217.08 & 0.0533 & 55 & 0.478 & & & $\mathrm{t}, \mathrm{p}$ \\
\hline
\end{tabular}

Notes. $\dagger$ : uncertain ID ( $>10 \%$ off sequence). c: possible combination frequency. $\mathrm{d}$ : doublet component. p: non-prewhitenable peak. t: tentative mode (between 4 and $\left.4.2 \sigma_{\mathrm{FT}}\right)$. u: unidentifiable peak.

is slightly longer than the longest spacing detected in the 13 V1093 Her stars observed by Kepler and studied by Reed et al. (2011, 2012), of $271.15 \mathrm{~s}$, and associated with $\ell=1$ modes. For all but two of those stars a second sequence was found to

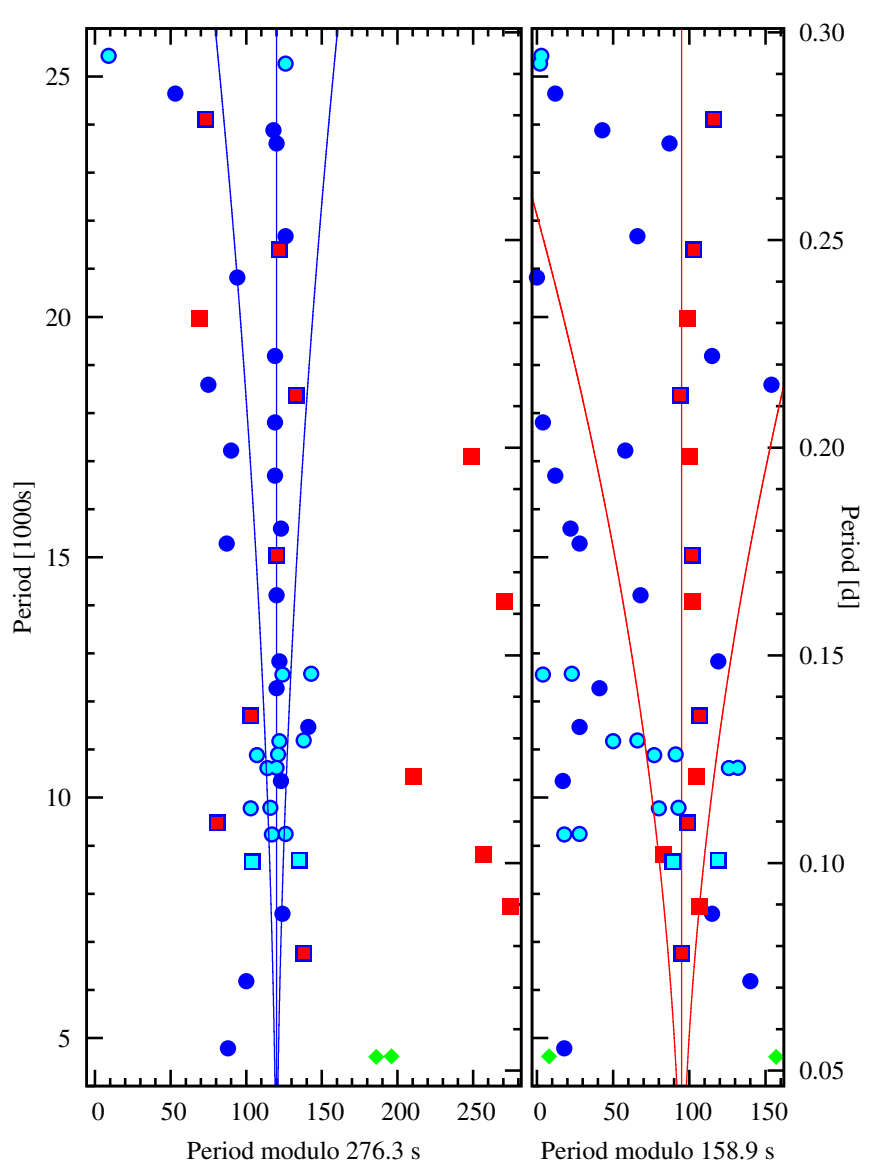

Figure 3. Échelle diagram for KIC 1718290. Bullets indicate $\ell=1$ modes (blue in the online version) and boxes $\ell=2$ modes (red). Periods that may belong to either sequence are marked with outline boxes (red core with blue outline). Periods which are part of doublets are marked with hollow bullets or boxes (blue with cyan cores), for $\ell=1$ or $\ell=2$ modes, respectively. Two points above $26,000 \mathrm{~s}$ (not shown) and two points below $5000 \mathrm{~s}$ (green diamonds) do not fit either sequence. The curves indicate the width of an $\ell=1$ triplet (left panel) and of an $\ell=2, m=-2,0,+2$ triplet (right panel), in the absence of any trapping. (A color version of this figure is available in the online journal.)

correspond to $\ell=2$ modes with a spacing $1 / \sqrt{3}$ times that of the $\ell=1$ sequence, exactly as predicted by theory. But whereas the $\ell=1$ sequence in KIC 1718290 is clearer than in any of these $13 \mathrm{~V} 1093 \mathrm{Her}$ pulsators, the $\ell=2$ sequence which should be at $\Delta \Pi_{2}=159.5$ is tentative at best. While an unusually large number of modes fall on the $276.3 \mathrm{~s}$ sequence within $\pm 1 \%$ $(14 / 54)$, a much larger number of modes fits within a more generous $\pm 10 \%$ interval $(35 / 54)$. Some of the modes appear to be doublets, including some of the strongest modes such as $f_{34,35}$. The same frequency splitting is seen in the pairs $f_{27,28}$, $f_{32,33}, f_{34,35}, f_{36,37}, f_{40,41}$, and $f_{43,44}$ (splittings between 0.115 and $0.125 \mu \mathrm{Hz})$. The $f_{4,5}$ doublet has twice that $(0.25 \mu \mathrm{Hz})$ and the $f_{46,47}$ doublet has three times the splitting $(0.39 \mu \mathrm{Hz})$. As the majority of these doublets fit the $\ell=1$ period spacing sequence, a possible interpretation is that they are the $m= \pm 1$ components of $\ell=1$ triplets. Unless one can invoke some other mechanism that would systematically suppress either the $\ell=$ -1 or the $\ell=+1$ component so that one never sees any triplets, the most plausible explanation is that the viewing angle is aligned with the pulsation equator, where the $m=0$ components suffer large cancellation. Following this interpretation, the $\ell=$ 2 modes which suffer the least geometric cancellation at this inclination are the $m= \pm 2$ components. Using $C_{n 1}=0.5$ and 
$C_{n 2}=0.16$, as indicated by the asymptotic theory, the $\ell=2$, $m= \pm 2$ components would be split by $\sim 0.40 \mu \mathrm{Hz}$ which fits the $f_{46,47}$ doublet. If we accept this argument and take $0.12 \mu \mathrm{Hz}$ to be the rotationally induced frequency splitting for an $\ell=1$, $m= \pm 1$ pair, then the rotation period is $\sim 100$ days. A similar rotationally induced splitting was found for the sdBV+WD binary KIC $11558725(0.13 \mu \mathrm{Hz}$; Telting et al. 2012). Note that the $\ell=1, m= \pm 1$ modes will impose a scatter in the échelle diagram that increases with increasing period, as indicated by the curves in Figure 3 . For the $f_{4,5}$ doublet the splitting is $\sim 100 \mathrm{~s}$, making secure identification by sequence spacing rather hopeless. As can be seen in the left panel of Figure 3, only five modes cannot be fitted on a generous $\ell=1$ sequence, when tentative peaks and the long-period modes $f_{2,3}$ are ignored. If we impose a sequence at $\Delta \Pi_{2}=158.9 \mathrm{~s}$, the remaining five modes can also be explained, and we see that seven modes fit both sequences. The $\ell=2$ sequence is far from convincing, but forthcoming Kepler data should reduce the noise and may substantiate it. We performed linear-regression fitting to the identified periods and a Kolmogorov-Smirnov (K-S) test as in Reed et al. (2011). The fits to the period spacings were 276.3(1) and $158.91(5) \mathrm{s}$ with K-S significances of $6.2 \sigma$ and $0.98 \sigma$ for the $\ell=1$ and $\ell=2$ sequences, respectively.

\section{DISCUSSION}

The pulsation spectrum of KIC 1718290 shows the same richness and characteristic period spacings as that of the 13 V1093 Her pulsators monitored by Kepler, with the exception that the pulsations have longer periods (by about a factor of two).

We constructed a MESA model for the Sun (Paxton et al. 2011) and evolved it from the pre-main-sequence phase up to the point of core-helium ignition, assuming standard Reimers mass loss (Reimers 1975; with $\eta=0.5$ ). Just before helium ignition we stripped the stellar model of most of its envelope, leaving only a residual hydrogen envelope on top of the He-core. The model was then relaxed to a new equilibrium state. Different models with varying thickness of the $\mathrm{H}$-layer were then evolved through the He-flashes, the He-core-burning and He-shellburning stages. Note that these models were computed with default MESA parameters, and do not reproduce the position of the observed EHB very well. A number of model parameters can be tweaked to change the position of the EHB. For instance, it is very sensitive to the exact mass where the helium flash occurs. As shown by Dorman et al. (1993), increasing the helium abundance and/or reducing the metallicity would shift it in the right direction, but since we have no reason to assume unconventional initial abundances that apply to all progenitors of $\mathrm{BHB} / \mathrm{EHB}$ stars, we decided to leave the offset as it is for now.

In Figure 4 we show two extended tracks from this set, and six tracks truncated to show just the core-burning stage where the stars spend most of their time. The short-lived stages prior to stable core-helium burning and after the end of shell-helium burning are shown with thin lines. The $\sim 80$ Myr core-heliumburning stages are plotted with thick lines, and the $\sim 62 \mathrm{Myr}$ shell-helium-burning stages are plotted with intermediately thick lines. The high-gravity evolutionary sequence $(M=$ $\left.0.465 M_{\odot}\right)$ is typical for an EHB star, while the lower-gravity sequence $\left(M=0.476 M_{\odot}\right)$ is more representative of the observed position of KIC 1718290. EHB stars evolve directly to the whitedwarf cooling curve after ending their helium burning, while normal $\mathrm{HB}$ stars ascend the AGB. The $M=0.476 M_{\odot}$ model

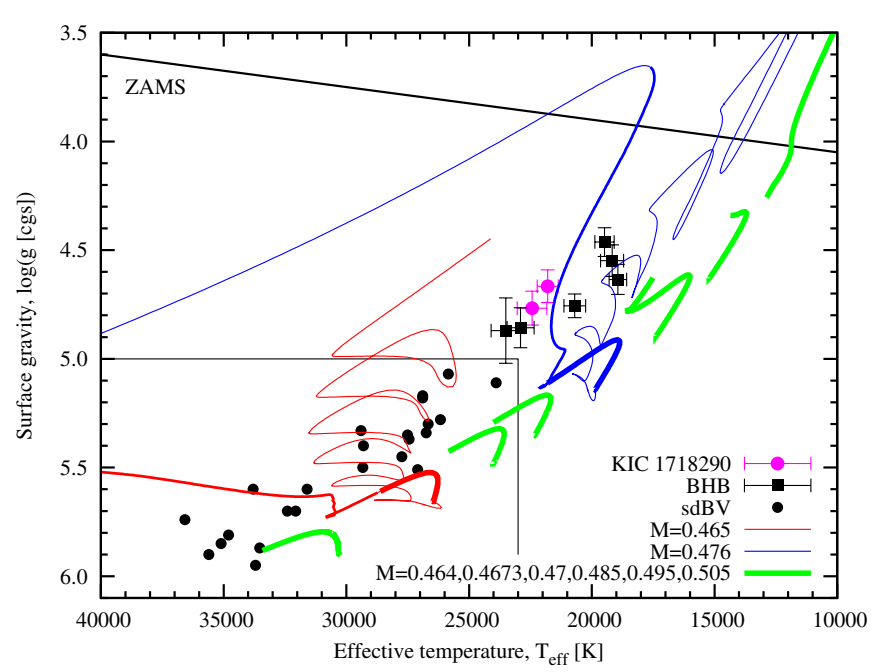

Figure 4. $T_{\text {eff }}-\log g$ plane with evolutionary models and some sample stars. The target and six other intermediate-helium stars are plotted with error bars indicating three times the formal-fitting errors. The parameters from the WHT spectrum and the NOT spectrum are shown as bullets with error bars. The classical EHB region is outlined, and encompasses a number of sdB pulsators that we have fitted on the same model grid as KIC 1718290.

(A color version of this figure is available in the online journal.)

displays an intermediate behavior, where its radius expands substantially and the temperature drops slightly, but still never getting close to the AGB, just barely reaching the $T_{\text {eff }} / \log$ $g$-level of the main sequence before faltering and starting the contraction that will eventually take it to the white-dwarf cooling curve.

In Figure 4 the location of the target and six other intermediate-helium stars, PG 0229+064, PG 0848+186, PG 1400+389, PG 2356+167, Balloon 82000001, and CPD-20 1123 , are shown. CPD-20 1123 was recently shown to be a binary with an orbital period of 2.3 days (Neelamkodan et al. 2012). For the other stars, no information about binarity has been published. All these stars have $\log N_{\mathrm{He}} / N_{\mathrm{H}}$ between -1.0 and -0.5 , i.e., they all have somewhat super-solar helium abundances. Most stars classified as He-sdB and He-sdO are extremely helium rich; their photospheres have more helium than hydrogen by number, sometimes by a factor of $10^{3}$, and may be associated with double-helium-white-dwarf mergers (Zhang \& Jeffery 2012). The intermediate-helium sdB stars appear confined to a more narrow region of the $T_{\text {eff }} / \log g$ plane than the extreme-helium sdBs are, implying a narrower core-mass range.

At higher gravities in Figure 4, the pulsating stars from the Kepler sample are plotted with their physical parameters from Østensen et al. (2010c, 2011b), and further down the EHB we include some V361 Hya pulsators from Østensen et al. (2010b) that were fitted on the same LTE grid as the other stars. Note that the number of stars marked in Figure 4 is in no way representative of the population densities of these stars. The EHB stars in the figure are just 24 out of more than 2000 known sdBs (Østensen 2006), while the BHB stars indicated include the majority of stars similar to KIC 1718290 described in the literature. Thus, as pointed out by Saffer et al. (1994, 1997), what is known as the second Newell gap from studies of globular clusters (Newell \& Graham 1976) is neither a forbidden region on the BHB nor due to selection effects, but rather a very underpopulated region that coincides with the position where the envelope has the critical mass required for hydrogen-shell 
burning. Explaining the exact process that produces the gap remains a challenge. As one proceeds from this gap toward the redder part of the $\mathrm{HB}$, it becomes exceedingly hard to distinguish between field-HB-B stars and main-sequence-B stars, but highresolution spectroscopy can distinguish their peculiar chemical composition and low rotation rates (Hambly et al. 1997).

\section{CONCLUSIONS}

We have shown that KIC 1718290 is spectroscopically an intermediate-helium-sdB star, most likely associated with the BHB. Photometric data of extremely high precision and coverage obtained with Kepler have revealed a pulsation spectrum with a clear period spacing of 276.3 s, indicative of a corehelium-burning star similar to the V1093 Her pulsators. There is no reason to believe that the driving mechanism responsible for exciting the pulsations in KIC 1718290 is any other than the same $\kappa$-mechanism that drives the pulsations in the V1093 Her and V361 Hya stars (Fontaine et al. 2003). The red edge of the V1093 Her instability strip has never been firmly established, due to the sharp drop in the number of observed EHB stars at around 24,000 K. V1093 Her pulsations in stars at the blue tip of the BHB should not really come as a big surprise, as early models for these stars predicted pulsations at the cool end of the EHB and beyond (Jeffery \& Saio 2006).

KIC 1718290 must have suffered extreme mass loss during its evolution in order to reach its present configuration. While our spectral energy distribution (SED) fit displays no sign of any main-sequence companion, a white-dwarf companion is not excluded. The Kepler photometry rules out any close object, since a period of 10 days or less would be readily detected from Doppler beaming in the light curve, unless the orbit is seen face on (Telting et al. 2012). White-dwarf companions with orbits of $\sim 30$ days are harder to detect with Kepler due to the monthly data-download cycle, and the reacquisition of the field after each such maneuver introduces trends in the data that must be filtered out. Spectroscopic follow-up observations are being undertaken, but have not yet provided any limits stronger than can be determined from photometry.

We conclude that we have observed $g$-mode pulsations in a star at the blue end of the classical HB that are driven by the same $\kappa$-mechanism as in the V1093 Her pulsators and slowly pulsating main-sequence-B stars. The pulsations are of longer period and lower maximum amplitude than has been observed in any of the Kepler V1093 Her targets, making this type of pulsator practically undetectable with ground-based observations. In analogy with the naming sdBV for the group consisting of V361 Hya and V1093 Her pulsators, we propose to call KIC 1718290 and similar BHB variables BHBV stars. The rarity of $\mathrm{BHB}$ stars at similar temperatures will make it hard to find other examples of such pulsators in the limited field of view of Kepler. However, the question of how far toward the red along the BHB $g$-mode pulsations may extend remains a question to be explored.

The authors gratefully acknowledge the Kepler team and everybody who has contributed to making this mission possible.
Funding for the Kepler Mission is provided by NASA's Science Mission Directorate. Spectroscopic observations were made with the William Herschel Telescope located at the Observatorio del Roque de los Muchachos (ORM), the Nordic Optical Telescope also at the ORM, and the Steward Observatory $2.3 \mathrm{~m}$ Bok telescope. This research has received funding from the European Research Council under the European Community's Seventh Framework Programme (FP7/2007-2013)/ERC grant No. 227224 (PROSPERITY), as well as from the Research Council of KU Leuven grant GOA/2008/04.

\section{REFERENCES}

Baran, A. S., Kawaler, S. D., Reed, M. D., et al. 2011, MNRAS, 414, 2871 Borucki, W. J., Koch, D. G., Basri, G., et al. 2011, ApJ, 736, 19 Christy, R. F. 1966, ApJ, 144, 108

Degroote, P., Acke, B., Samadi, R., et al. 2011, A\&A, 536, A82

Dorman, B., Rood, R. T., \& O'Connell, R. W. 1993, ApJ, 419, 596

Fontaine, G., Brassard, P., Charpinet, S., et al. 2003, ApJ, 597, 518

Gilliland, R. L., Brown, T. M., Christensen-Dalsgaard, J., et al. 2010, PASP, 122,131

Green, E. M., Fontaine, G., Reed, M. D., et al. 2003, ApJ, 583, L31

Hambly, N. C., Rolleston, W. R. J., Keenan, F. P., Dufton, P. L., \& Saffer, R. A. 1997, ApJS, 111, 419

Han, Z., Podsiadlowski, P., Maxted, P. F. L., \& Marsh, T. R. 2003, MNRAS, 341,669

Han, Z., Podsiadlowski, P., Maxted, P. F. L., Marsh, T. R., \& Ivanova, N. 2002, MNRAS, 336, 449

Hansen, C. J., Winget, D. E., \& Kawaler, S. D. 1985, ApJ, 297, 544

Heber, U. 1986, A\&A, 155, 33

Heber, U., Reid, I. N., \& Werner, K. 2000, A\&A, 363, 198

Jeffery, C. S., \& Saio, H. 2006, MNRAS, 371, 659

Kawaler, S. D., Reed, M. D., Østensen, R. H., et al. 2010a, MNRAS, 409, 1509

Kawaler, S. D., Reed, M. D., Quint, A. C., et al. 2010b, MNRAS, 409, 1487

Kilkenny, D., Koen, C., O’Donoghue, D., \& Stobie, R. S. 1997, MNRAS, 285 , 640

Kurucz, R. L. 1993, VizieR Online Data Catalog, 6039, 0

Neelamkodan, N., Geier, S., Jeffery, C. S., et al. 2012, MNRAS, in press (arXiv:1204.4387)

Newell, B., \& Graham, J. A. 1976, ApJ, 204, 804

Østensen, R. H. 2006, Balt. Astron., 15, 85

Østensen, R. H. 2009, Commun. Asteroseismol., 159, 75

Østensen, R. H., Bloemen, S., Vučković, M., et al. 2011a, ApJ, 736, L39

Østensen, R. H., Green, E. M., Bloemen, S., et al. 2010a, MNRAS, 408, L51

Østensen, R. H., Oreiro, R., Solheim, J., et al. 2010b, A\&A, 513, A6

Østensen, R. H., Silvotti, R., Charpinet, S., et al. 2010c, MNRAS, 409, 1470

Østensen, R. H., Silvotti, R., Charpinet, S., et al. 2011b, MNRAS, 414, 2860

Paxton, B., Bildsten, L., Dotter, A., et al. 2011, ApJS, 192, 3

Prša, A., Guinan, E. F., Devinney, E. J., \& Engle, S. G. 2008, A\&A, 489, 1209

Ramspeck, M., Heber, U., \& Edelmann, H. 2001, A\&A, 379, 235

Reed, M., Kawaler, S. D., Østensen, R. H., et al. 2010, MNRAS, 409, 1496

Reed, M. D., Baran, A., Quint, A. C., et al. 2011, MNRAS, 414, 2885

Reed, M. D., Baran, A. S., Quint, A. C., et al. 2012, in ASP Conf. Ser. 452, The Fifth Meeting on Hot Subdwarf Stars and Related Objects, ed. D. Kilkenny, S. Jeffery, \& C. Koen (San Francisco, CA: ASP), 185

Reimers, D. 1975, Mem. Soc. R. Sci. Liege, 8, 369

Saffer, R. A., Bergeron, P., Koester, D., \& Liebert, J. 1994, ApJ, 432, 351

Saffer, R. A., Keenan, F. P., Hambly, N. C., Dufton, P. L., \& Liebert, J. 1997, ApJ, 491, 172

Schlegel, D. J., Finkbeiner, D. P., \& Davis, M. 1998, ApJ, 500, 525

Stoughton, C., Lupton, R. H., Bernardi, M., et al. 2002, AJ, 123, 485

Telting, J. H., Østensen, R. H., Baran, A. S., et al. 2012, A\&A, submitted

Zhang, X., \& Jeffery, C. S. 2012, MNRAS, 419, 452 